\title{
Stratified-Sampling Estimation of Outage Probability in Nonlinear Coherent Systems with Polarization Dependent Loss
}

\author{
N. Rossi, P. Serena, A. Bononi
}

Università degli Studi di Parma, Dipartimento di Ingegneria dell'Informazione, v.le G. Usberti 181/A, 43124 Parma (Italy), $\bowtie$ rossi_n@tlc.unipr.it

\begin{abstract}
A novel Stratified-Sampling algorithm is used to estimate the outage probability due to nonlinearity, PDL and PMD in a 15-channel 32Gbaud PDM-QPSK system over a 30×100km dispersionmanaged NZDSF link.
\end{abstract}

\section{Introduction}

Early studies on coherent systems assessed the impact of polarization dependent loss (PDL) in the linear regime ${ }^{1,2}$, where PDL induces both a degradation of the signal to noise ratio (SNR) and a loss of orthogonality between polarization tributaries. Recently, interest has arisen in the interplay between PDL and fiber nonlinearity $(\mathrm{NL})$, which leads to an average Q-factor degradation ${ }^{3,4}$. However, this interplay also affects the tails of the Q-factor distribution ${ }^{5,6}$, thus affecting the outage probability (OP). Thorough OP estimation accounting for fiber NL is a time-consuming, challenging task both in lab experiments with offline Q-factor estimation and in Monte-Carlo (MC) based simulations.

In this paper, for the first time, we present OP curves versus PDL for a coherent wavelength division multiplexed (WDM) dispersion-managed (DM) system in presence of Kerr nonlinearity, $\mathrm{PDL}$, and polarization mode dispersion (PMD). A new stratified-sampling (SS) algorithm is introduced to more than double the efficiency with respect to standard MC estimation and thus make the simulations feasible.

\section{Numerical Setup}

We simulated with the open-source software Optilux the transmission of 15 WDM channels with 32Gbaud non-return to zero (NRZ) polarization division multiplexing quadrature phase shift keying (PDM-QPSK) modulation (including $23 \%$ overhead) over a $30 \times 100 \mathrm{~km}$ DM link, with non-zero dispersion-shifted (NZDSF) transmission fiber (dispersion $4 \mathrm{ps} / \mathrm{nm} / \mathrm{km}$, attenuation $0.2 \mathrm{~dB} / \mathrm{km}$, nonlinear index $\gamma=1.51 / \mathrm{W} / \mathrm{km}$ ). The channel spacing was $50 \mathrm{GHz}$. The in-line residual dispersion was $30 \mathrm{ps} / \mathrm{nm} / \mathrm{span}$, the precompensation was $-495 \mathrm{ps} / \mathrm{nm}$, and the overall dispersion was set to zero by the receiver dispersion equalizer. We assumed zero carrier-local oscillator frequency offset, and zero laser phase noise. At the receiver, coherent detection of the central channel used a digital signal processor (DSP) including: analog to digital conversion (ADC) with bandwidth $17 \mathrm{GHz}, 2$ samples-persymbol data-aided least squares butterfly equalization with 7 taps, and Viterbi and Viterbi (V\&V) phase estimation with 15 taps.

Fiber propagation used the split-step Fourier algorithm (SSFA) applied to the Manakov-PMD equation. Each channel was modulated by 1024 purely random symbols within the SSFA time window. Flat-gain amplifiers $(6 \mathrm{~dB}$ noise figure) added inline amplified spontaneous emission (ASE) noise thus accounting for nonlinear signalnoise interactions. PDL was emulated by lumped elements placed before the transmission fibers.

To correctly estimate the OP, it is fundamental to distinguish between random variables $(R V)$ that do not vary during each Q-factor measurement (non-ergodic) and those that do (ergodic). Non-ergodic RVs were: carrier input state of polarization, orientation of PDL elements and PMD waveplates. Ergodic RVs were: symbol patterns and ASE. OP estimation requires two nested estimations: 1) an inner $Q$-factor estimation on ergodic RVs: for each realization of non-ergodic $\mathrm{RVs}$, the BER (then converted to Q-factor) was MC-estimated by iterating over the ergodic RVs until 400 errors were counted; 2) an outer OP estimation: we iterated over non-ergodic RVs, an outage was counted when the Q-factor was below the forward error correction (FEC) threshold of $6.25 \mathrm{~dB}$, and the outer estimation was stopped when at least 20 outages were counted.

\section{Stratified-Sampling Monte-Carlo Algorithm}

For the outer estimation, we propose using SS, a form of importance sampling where independent $M C$ estimations are performed over disjoint subsets, or strata, of the input sample space ${ }^{7}$. The crucial point of SS is the choice of a suitable space partition. Ideally, SS requires that in 


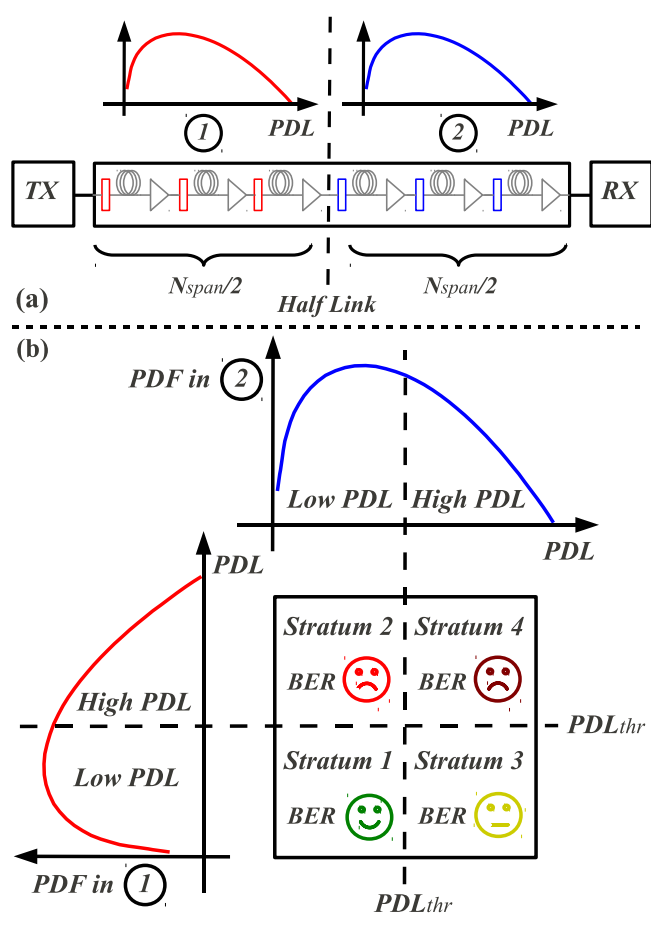

Fig. 1: Sketch of SS idea. The link is split in two halves (a). The PDL in each half follows a distribution whose median is the threshold that we use to discern between "low" and "high" PDL. This choice identifies four strata (b). Accordingly to SS theory, running one MC simulation within each stratum is faster than one global MC simulation, see text.

each stratum the observed RV has small (ideally zero) variance, such that running an $\mathrm{MC}$ sampling in a stratum requires just a few (ideally just one) samples. In our case the input space is represented by all possible PDL configurations and the observed RV is the indicator of the event " $Q$-factor below FEC threshold".

Our novel idea is based on the following observations. In linear propagation, PDL degrades more the SNR (hence Q-factor) if it takes place at the beginning of the link, according to Friis formula. On the other hand, in presence of NL the most detrimental effect of PDL is the generation of peak power variations ${ }^{4}$. If these variations take place early along the link, they accumulate a large nonlinear phase in the remaining part of the link, thus, again, degrading performance.

These observations suggest to stratify the sample space in terms of the way PDL builds up along the link. We operate as sketched in Fig. 1. We first generate a random realization of PDL elements. We then measure the cumulated PDL in both the first and the second half of the link. Comparing such values with a chosen threshold $P D L_{t h r}$, we discern between "low" and "high" PDL cumulated in the half of interest. This deci- sion corresponds to identifying a stratum in the sample space. For the sake of simplicity we chose $P D L_{t h r}$ equal to the median. We thus have four strata that we separately visit by four $\mathrm{MC}$ simulations. Optimal SS visits each stratum with a frequency proportional to the variance of the observed RV within the stratum ${ }^{7}$. The frequency of visit is regulated by an accept/reject test after generating a random PDL configuration. Once a particular PDL realization is accepted, i.e., the stratum is identified, we run the SSFA as described in Sec. 2. The simulation is stopped when the OP is estimated with the desired accuracy ${ }^{7}$.

\section{Numerical Results}

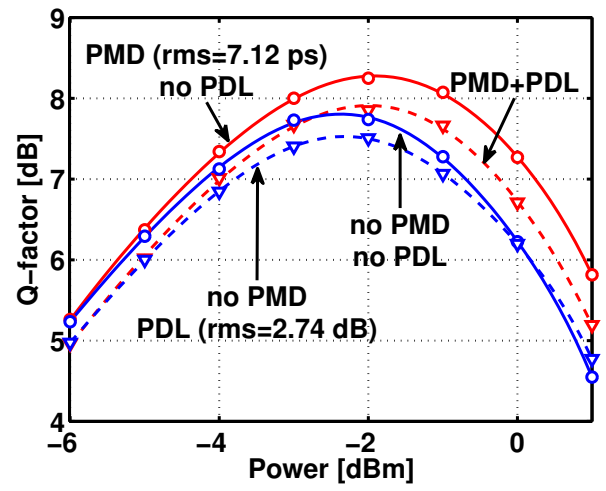

Fig. 2: Average Q-factor vs power for a 15-channel homogeneous 32Gbaud PDM-QPSK system over a DM $30 \times 100 \mathrm{~km}$ NZDSF link.

Average Q-factor. Fig. 2 shows the Q-factor of the reference center channel averaged over 10 realizations of non-ergodic RVs versus input power. Average $Q$ was obtained with standard MC. We observe that in absence of PMD, the best average $\mathrm{Q}$-factor is reduced by $0.3 \mathrm{~dB}$ in presence of a root-mean-square (rms) PDL of 2.74 $\mathrm{dB}$. It is worth noting that the PDL detrimental effect on average $Q$ vanishes in the strongly nonlinear regime. This is due to the partial polarization induced by PDL that reduces cross-polarization modulation (XPolM), which is the dominant $\mathrm{NL}$ in this kind of links ${ }^{8}$. The best $Q$ improves by about $0.4 \mathrm{~dB}$ in presence of a typical PMD of $0.13 \mathrm{ps} / \sqrt{\mathrm{km}}$. In DM links, PMD reduces NL because of its split-and-delay and depolarizing effects ${ }^{9}$. With PMD, however, the extra PDL penalty on average $Q$ remains almost constant with the input power. For the same system with $P M D=0$, we next concentrated the link PDL into a block of 5 consecutive spans with aligned PDL elements. Fig. 3 shows average $Q$ factor versus the starting span $x$ of this block. Cumulated PDL is indicated 


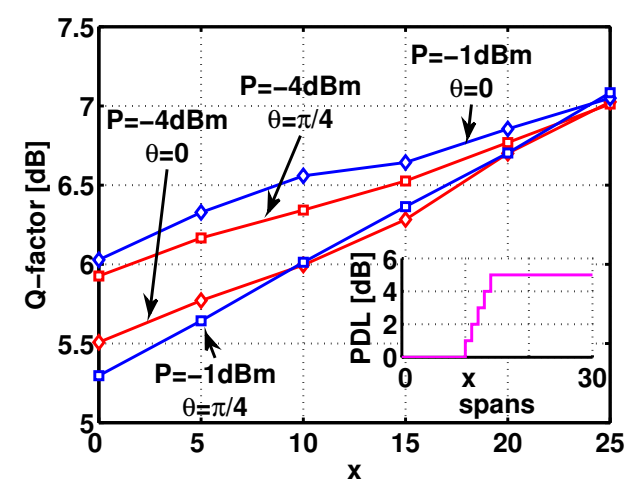

Fig. 3: Average Q-factor vs starting span $x$ of a 5-span block containing the entire line PDL of $5 \mathrm{~dB}$. Red: linear; Blue: nonlinear regime. $\mathrm{PMD}=0 . \theta$ is Stokes angle of the main PDL eigenvector.

in the inset of Fig. 3. $\theta$ is the Stokes angle between the main PDL eigenvector and tributary $X$ of reference channel ${ }^{3}$. We can see that both in the linear $(P=-4 \mathrm{dBm}$, red curves) and nonlinear $(P=-1 \mathrm{dBm}$, blue curves) regimes the early presence of PDL along the link is most detrimental, in agreement with the intuition on stratification in our SS. Also, $\theta=0$ is most detrimental in linear regime, while $\theta=\pi / 4$ in NL regime, in agreement with ${ }^{3}$.

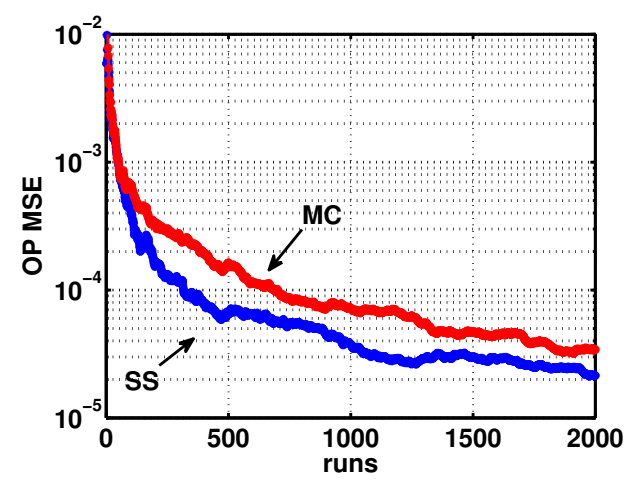

Fig. 4: OP Mean Square Error vs number of runs for $\mathrm{MC}$ and SS algorithms, at a true $O P=6 \cdot 10^{-2}$, rms $\mathrm{PDL}=4.4 \mathrm{~dB}$. Linear propagation without PMD.

Outage Probability. We first evaluated the OP in a simple case where MC was feasible, in order to compare SS and MC efficiency. We used an input power $P=-1.5 \mathrm{dBm}, \mathrm{PDL}=4.4 \mathrm{~dB}$, no PMD and no NL. With MC, we took $80,000 \mathrm{Q}$ samples, divided into 40 parallel simulations of 2000 samples each. The MC-estimated average on all samples $O P=6 \cdot 10^{-2}$ was declared the true value. While the 40 simulations were progressing from 1 to 2000 runs (samples), we also measured the running OP estimate, and calculated the OP mean-square error (MSE). We then ran 40 SS simulations and again estimated the MSE. Fig. 4 gives the OP MSE versus the number of collected $Q$ samples. We note that SS allows a saving of more than $50 \%$ in runs compared to $\mathrm{MC}$ at the same MSE level.

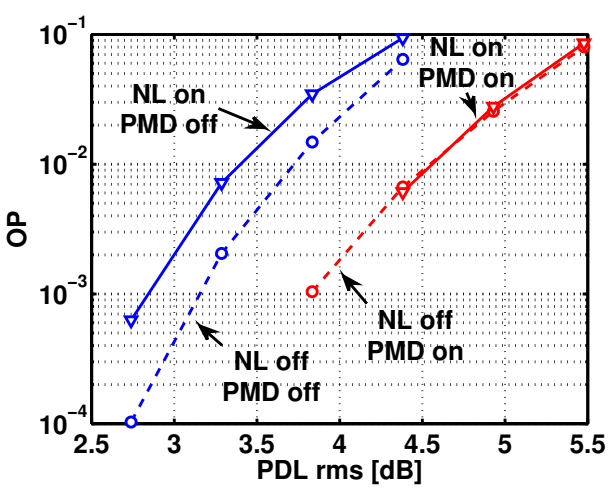

Fig. 5: Outage probability vs PDL. 15-channel homogeneous 32Gbaud PDM-QPSK system over a DM $30 \times 100 \mathrm{~km}$ NZDSF link. $P=-1.5 \mathrm{dBm}$.

Fig. 5 shows in solid lines the SS-estimated $\mathrm{OP}$ versus PDL, at rms PMD both zero and 7.12 ps and power $P=-1.5 \mathrm{dBm}$. Dashed lines show the OP of the "equivalent" system without NL but with increased noise figure such that at zero $P D L$ the average $\mathrm{Q}$-factor is same as with $\mathrm{NL}$, i.e. $Q_{a v g}=7.6 \mathrm{~dB}$ at $\mathrm{PMD}=0$ and $Q_{a v g}=8.2 \mathrm{~dB}$ at $\mathrm{PMD}=7.12 \mathrm{ps}$ (Cfr. solid lines at $P=-1.5 \mathrm{dBm}$ in Fig. 2). As we add PDL, if NL and ASE were equally affected by PDL, the solid and dashed curves would coincide. This is not the case in absence of PMD. In presence of typical PMD, instead, simulations show that OP in the NL system are close to those in the equivalent linear system, at least at $O P>5 \cdot 10^{-3}$.

\section{Conclusions}

We proposed a stratified-sampling algorithm to estimate the outage probability in nonlinear coherent systems with PDL. Results confirm that PDL and NL do have significant interactions. At typical PMD values, simulations at large OP suggest that OP due to PDL could be estimated on an equivalent linear system. More points at lower $\mathrm{OP}$ are required to confirm such a fact, but these require longer and longer simulation times.

\section{References}

1 M. Shtaif, Opt. Expr., 16, 13918 (2008).

2 C. Xie, Proc. OFC'10, OWE6 (2010).

3 O. Vassilieva et al., Proc. ECOC'10, P4.08 (2010).

4 Z. Tao et al., Proc. OECC'11,6B3_2 (2011).

5 O. Vassilieva et al., Proc. ECOC'12, We3C4 (2012).

6 O. Vassilieva et al., Proc. OFC'13, OM3B.6 (2013).

7 P. Serena et al., J. Lightw. Technol. 27, 2404 (2009).

8 A. Bononi et al., Proc. ECOC'10, Th.10.E.1 (2010).

9 P. Serena et al., J. Lightw. Technol. 29, 1691 (2011). 